\title{
Towards genomic-based prognostication and precision therapy for diffuse large B-cell Iymphoma
}

\author{
Marthe Minderman ${ }^{1,2}$ and Steven T. Pals ${ }^{1,2}$ \\ ${ }^{1}$ Department of Pathology, Cancer Center Amsterdam, Amsterdam UMC, University of Amsterdam and 'Lymphoma and Myeloma \\ Center Amsterdam - LYMMCARE, Amsterdam, the Netherlands.
}

E-mail: STEVEN T. PALS - s.t.pals@amsterdamumc.nl

doi:10.3324/haematol.2020.255448

T he development of precision medicine for diffuse large B-cell lymphoma (DLBCL) is complicated by its great clinical and molecular heterogeneity. Twenty years ago, two distinct cell-of-origin (COO) subtypes were identified based on distinct gene expression profiles that reflect different stages of B-cell development, i.e., germinal center B-cell-like (GCB) and activated B-celllike $(A B C) D L B C L .{ }^{1}$ The ongoing revolution in genomics has since shed light on the genetic landscape of these subtypes. Whereas ABC DLBCL is characterized by frequent mutations in nuclear factor- $\kappa \mathrm{B}(\mathrm{NF}-\kappa \mathrm{B})$ pathway drivers and intermediates, including TNFAIP3, MYD88, CARD11 and $C D 79 B$, as well as loss of cell cycle regulators $C D K N 2 A$ and $C D K N 2 B, G C B$ DLBCL carry frequent mutations in epigenetic modifiers, such as CREBBP, KMT2D and EZH2.,3 GCB DLBCL patients have a more favorable prognosis and a better clinical response to standard R-CHOP immunochemotherapy than those with ABC DLBCL. Nevertheless, the clinical outcome is heterogenous in both subtypes with an unfavorable outcome in a substantial proportion of patients, also in individuals with GCB DLBCL. ${ }^{1,4}$ Currently, the impact of somatic mutations and other genomic aberrations on the clinical outcome and therapy response is still not completely understood and there is a high need for targeted precision therapy.

In this issue of Haematologica, Bolen et al. report the frequency and prognostic impact of genomic alterations in 499 untreated DLBCL patients enrolled in the GOYA study (clinicaltrials.gov identifier: NCT01287741). ${ }^{5}$ Using a well-validated targeted next-generation sequencing (NGS) approach, the authors demonstrate that only alterations of the BCL2 gene, translocations as well as single nucleotide variants (SNV), were significantly associated with reduced progression-free survival (PFS), independent of other molecular or clinical factors, including $\mathrm{COO}$ or the International Prognostic Index (IPI). In line with previous studies, BCL2 was the most frequently mutated gene in GCB DLBCL and there was a strong correlation between a BCL2 translocation and presence of BCL2 mutations, which presumably are a consequence of aberrant somatic hypermutation., While $B C L 2$ translocations and SNV were highly enriched in the GCB subtype, BCL2 gene amplifications were more frequently detected in the ABC subtype. The findings suggest that a select subset of DLBCL patients may likely benefit from pharmacological inhibition of BCL2, as an addition to standard immunochemotherapy. The highly selective BCL2 inhibitor venetoclax strongly improved PFS in chronic lymphocytic leukemia (CLL) patients and is currently under investigation for DLBCL.,8 Preliminary findings from the phase II CAVALLI trial indicate that addition of venetoclax to R-CHOP therapy can improve therapy outcome in patients with BCL2-positive lymphomas. The largest benefit was observed for BCL2-translocated and double-hit lymphomas, suggesting that the addition of venetoclax may be of particular value for these therapyresistant and aggressive subgroups.

Although the other genetic aberrations identified by Bolen et al. did not offer prognostic value over well-established risk factors, such as COO and IPI, they nevertheless provide biological insights that are crucial for the design of precision therapies (Figure 1). For example, in line with previous studies, the authors demonstrate that a large fraction of GCB DLBCL (16\%) carry gain-of-function mutations in the transcriptional repressor EZH2, implying that these patients could benefit from treatment with EZH2 inhibitors, such as tazemetostat. Indeed, preliminary results from a phase II clinical trial in 165 DLBCL and follicular lymphoma (FL) patients demonstrate that tazemetostat achieves favorable clinical responses in patients carrying activating EZH2 mutations. ' Likewise, the presence of inactivating mutations in the transcriptional activators KMT2D, CREBBP, EP300 and MEF2B implies that a subset of patients could benefit from treatment with histone deacetylase (HDAC) inhibitors. A phase II clinical trial evaluating the safety and therapeutic benefits of pan-HDAC inhibitor panobinostat showed durable responses in 11 out of 40 DLBCL patients, an effect that may be associated with mutations in transcriptional activator $M E F 2 B .{ }^{10}$ Activating mutations in $M E F 2 B$ are present in approximately $10 \%$ of DLBCL and FL patients and contribute to lymphomagenesis by enhancing the transcription of the BCL6 oncogene. ${ }^{11}$

Two independent landmark studies published in 2018 by Schmitz et al..$^{12}$ and Chapuy et al. ${ }^{13}$ have assessed the occurrence of somatic mutations, copy number alterations (CNA) and structural variants (SV) in a large cohort of DLBCL patients. Building on these observations, Wright et al. recently developed the 'LymphGen algorithm', which calculates the probability that a given tumor belongs to one of seven subtypes, based on its genetic features. ${ }^{14}$ The identified genetic subtypes are associated with differential responses to immunochemotherapy and, moreover, provide ample opportunity for the design of novel targeted (combination) treatments. In accordance with these and other previous studies, the study of Bolen et al..$^{5}$ reports that B-cell receptor (BCR) complex component CD79B and Toll-like receptor (TLR) adaptor protein MYD88 are among the most frequently mutated genes in ABC DLBCL. These mutations define the 'MCD' cluster of Wright et al., ${ }^{14}$ 


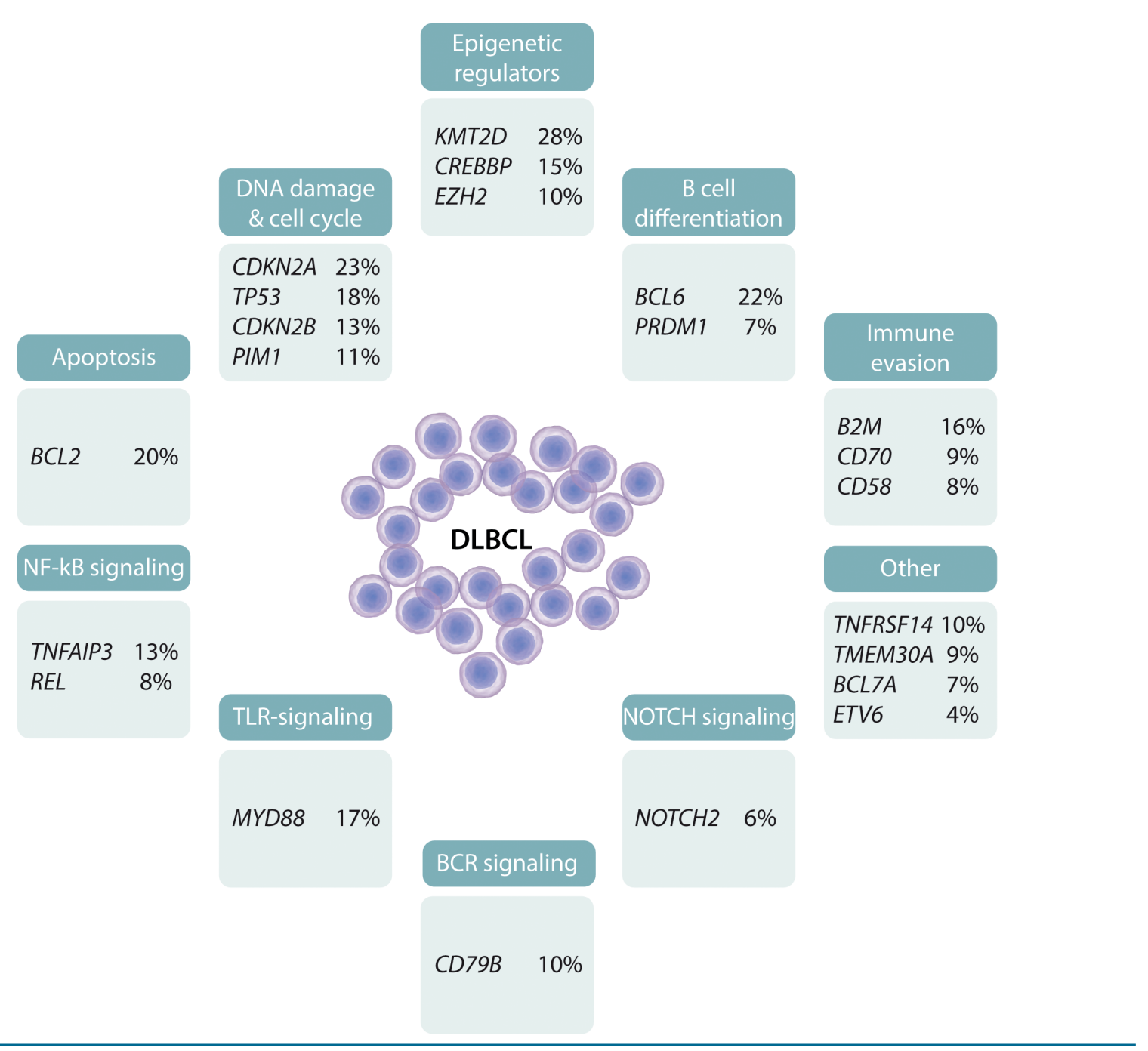

Figure 1. Prevalence of mutations in genes involved in various functional pathways in diffuse large B-cell lymphoma (DLBCL). Most frequently mutated genes as identified by Bolen et al. ${ }^{5}$ in a cohort of 482 DLBCL patients arranged according to the affected pathways. NF-KB: nuclear factor kappa B; TLR: Toll-like receptor; BCR: B-cell receptor.

comprising tumors characterized by a high prevalence and co-occurrence of MYD 88 and $C D 79 B$ mutations that are almost exclusively classified as ABC DLBCL and have an unfavorable prognosis. ${ }^{15}$ Intriguingly, this genetic subgroup is linked to primary extranodal lymphomas, including lymphomas arising in the central nervous system (CNS), ocular vitreo-retina and testis, all considered 'immune-privileged' sites as they tolerate allografts and permit only selective entrance of immune cells. ${ }^{16-19}$ Importantly, Wilson et al. established that $\mathrm{ABC} D L B C L$ harboring mutations in $C D 79 B$, particularly those with concurrent MYD 88 mutations, were highly responsive to treatment with ibrutinib, a selective Bruton's tyrosine kinase (BTK) inhibitor. ${ }^{20}$ These observations suggest that (extranodal) lymphomas belonging to the MCD subtype could also be targeted by inhibition of BCR signaling. Indeed, a phase Ib study in a panel of 18 primary central nervous system lymhpomas (PCNSL) demonstrated that ibrutinib monotherapy reduced tumor mass in $94 \%$ of patients. ${ }^{21}$ Additionally, a second phase I clinical trial showed clinical responses to ibrutinib in 10 out of 13 PCNSL patients, including five complete responses. ${ }^{22}$ Collectively, these suggest that patients with other primary extranodal lymphomas belonging to the MCD subtype, such as primary testicular or vitreoretinal lymphoma, might also benefit from treatment with $B C R$ pathway inhibitors.

In conclusion, the study by Bolen et al. ${ }^{5}$ fully confirms the previously described genetic heterogeneity and complexity of DLBCL. As a consequence of this complexity, well-established prognostic classifiers, such as $\mathrm{COO}$ and IPI, can only partially account for the differential responses to R-CHOP (and related) immunochemotherapy. The identification of alterations of the BCL2 gene as the only genetic abnormalities significantly associated with reduced PFS points towards targeting BCL2 as a rational addition to standard immunochemotherapy. Although not of (independent) prognostic value in the context of standard immunochemotherapy, genetic abnormalities defining potentially druggable targets/pathways were 
identified in a large proportion of the tumors across the distinct $\mathrm{COO}$ subtypes (Figure 1). Applying the publicly available LymphGen algorithm on the GOYA dataset could help classify patients into well-defined molecularly and clinically distinct subgroups. These newly characterized subsets can identify patients with an unfavorable prognosis and may guide the development of new precision therapies for these aggressive lymphomas.

\section{Funding}

Funded by a grant from LympheCo.

\section{References}

1. Alizadeh $\mathrm{AA}$, Eisen $\mathrm{MB}$, Davis RE, et al. Distinct types of diffuse large B-cell lymphoma identified by gene expression profiling. Nature. 2000;403(6769):503-511.

2. Pasqualucci L, Trifonov V, Fabbri G, et al. Analysis of the coding genome of diffuse large B-cell lymphoma. Nat Genet. 2011;43(9):830-837.

3. Lohr JG, Stojanov P, Lawrence MS, et al. Discovery and prioritization of somatic mutations in diffuse large B-cell lymphoma (DLBCL) by whole-exome sequencing. Proc Natl Acad Sci U S A. 2012;109(10):3879-3884

4. Rosenwald A, Wright G, Chan WC, et al. The use of molecular profiling to predict survival after chemotherapy for diffuse large-B-cell lymphoma. N Engl J Med. 2002;346(25):1937-1947

5. Bolen CR, Klanova M, Trneny M, et al. Prognostic impact of somatic mutations in diffuse large B-cell lymphoma and relationship to cellof-origin: data from the phase III GOYA study. Haematologica. 2020;105(9):2298-2307.

6. Schuetz JM, Johnson NA, Morin RD, et al. BCL2 mutations in diffuse large B-cell lymphoma. Leukemia. 2012;26(6):1383-1390.

7. Seymour JF, Kipps TJ, Eichhorst B, et al. Venetoclax-Rituximab in Relapsed or Refractory Chronic Lymphocytic Leukemia. N Engl J Med. 2018;378(12):1107-1120.

8. Morschhauser F, Feugier P, Flinn IW, et al. Venetoclax plus rituximab, cyclophosphamide, doxorubicin, vincristine and prednisolone (R$\mathrm{CHOP}$ ) improves outcomes in BCL2-positive first-line diffuse large B-cell lymphoma (DLBCL): first safety, efficacy and biomarker analyses from the phase II CAVALLI study. Blood. 2018;132 (Supplement 1):782.
9. Morschhauser F, Salles G, McKay P, et al. Interim Report from a Phase 2 Multicenter Study of Tazemetostat, an EZH2 Inhibitor: Clinical Activity and Favorable Safety in Patients with Relapsed or Refractory B-Cell Non-Hodgkin Lymphoma. Clin Lymphoma Myeloma Leuk. 2017;17(Suppl 2):S380-381.

10. Assouline SE, Nielsen TH, Yu S, et al. Phase 2 study of panobinostat with or without rituximab in relapsed diffuse large B-cell lymphoma. Blood. 2016;128(2):185-194.

11. Ying CY, Dominguez-Sola D, Fabi M, et al. MEF2B mutations lead to deregulated expression of the oncogene BCL6 in diffuse large B cell lymphoma. Nat Immunol. 2013;14(10):1084-1092.

12. Schmitz R, Wright GW, Huang DW, et al. Genetics and Pathogenesis of Diffuse Large B-Cell Lymphoma. N Engl J Med. 2018;378 (15):1396-1407.

13. Chapuy B, Stewart C, Dunford AJ, et al. Molecular subtypes of diffuse large B cell lymphoma are associated with distinct pathogenic mechanisms and outcomes. Nat Med. 2018;24(5):679-690.

14. Wright GW, Huang DW, Phelan JD, et al. A Probabilistic Classification Tool for Genetic Subtypes of Diffuse Large B Cell Lymphoma with Therapeutic Implications. Cancer Cell. 2020;37 (4):551-568.e14.

15. Vermaat JS, Somers SF, de Wreede LC, et al. MYD88 mutations identify a molecular subgroup of diffuse large B-cell lymphoma with an unfavorable prognosis. Haematologica. 2020;105(2):424-434.

16. Kraan W, Horlings HM, van Keimpema M, et al. High prevalence of oncogenic MYD88 and CD79B mutations in diffuse large B-cell lymphomas presenting at immune-privileged sites. Blood Cancer J. 2013;3(9):e139

17. Yonese I, Takase H, Yoshimori M, et al. CD79B mutations in primary vitreoretinal lymphoma: Diagnostic and prognostic potential. Eur J Haematol. 2019;102(2):191-196.

18. Schrader AMR, Jansen PM, Willemze R, et al. High prevalence of MYD88 and CD79B mutations in intravascular large B-cell lymphoma. Blood. 2018;131(18):2086-2089.

19. Kraan W, van Keimpema M, Horlings HM, et al. High prevalence of oncogenic MYD88 and CD79B mutations in primary testicular diffuse large B-cell lymphoma. Leukemia. 2014;28(3):719-720.

20. Wilson WH, Young RM, Schmitz R, et al. Targeting B cell receptor signaling with ibrutinib in diffuse large B cell lymphoma. Nat Med. 2015;21(8):922-926.

21. Lionakis MS, Dunleavy K, Roschewski M, et al. Inhibition of B Cell Receptor Signaling by Ibrutinib in Primary CNS Lymphoma. Cancer Cell. 2017;31(6):833-843.e5.

22. Grommes C, Pastore A, Palaskas N, et al. Ibrutinib Unmasks Critical Role of Bruton Tyrosine Kinase in Primary CNS Lymphoma. Cancer Discov. 2017;7(9):1018-1029.

\title{
Thrombin generation: a global coagulation procedure to investigate hypo- and hyper-coagulability
}

\author{
Armando Tripodi \\ IRCCS Ca' Granda Maggiore Hospital Foundation, Angelo Bianchi Bonomi Hemophilia and Thrombosis Center and Fondazione \\ Luigi Villa, Milano, Italy \\ E-mail: ARMANDO TRIPODI - armando.tripodi@unimi.it
}

doi:10.3324/haematol.2020.253047

$\mathrm{T}$ he article by van Paridon et al. ${ }^{1}$ published in this issue of Haematologica on results of thrombin generation (TG) in cardiovascular disease and mortality, stemming from the Gutenberg Health Study, provides an opportunity to comment on TG as a global laboratory procedure to investigate hypo- and hyper-coagulability.

TG as a laboratory test was developed in the early 1950s by McFarlane and Biggs ${ }^{2}$ and was based on the activation of coagulation in whole blood or plasma by triggers such as tissue factor or cephaline and calcium chlo- ride. The amount of thrombin generated over time was titrated by sampling the mixture at different time points into a fibrinogen solution and the resultant clotting times interpolated from a dose-response calibration curve to derive thrombin concentrations. Years later, Hemker et al. made substantial changes..$^{3-5}$ The fibrinogen solution was replaced by a chromogenic substrate specific for thrombin, test plasma was defibrinated prior to testing and computer software was developed to derive the parameters stemming from the TG curve. These changes made 